编者按: 课堂教学过程不仅是知识传递过程, 更是能力和素质养成过程。知识的传递和能力素质的养成, 都需要以 一定的教学理念为指导, 以一定的教学模式和方法做支撑。相较于知识传递, 能力和素质的养成对教学理念、教学 模式和方法的依存度更大: 同样一个知识点, 有的老师讲起来会清汤塞水、索然无味, 导致学生鼾声一片; 有的老 师讲起来则能启发兴趣、引发思考、激荡共鸣, 进而提升能力、启迪智慧。导致这种根本性差异的原因显然不在知 识本身, 而在于知识传递的理念、模式和方法。因此, “学科知识枯燥” 的判断是完全错误的, 是老师不懂得或者惰 于挖掘, 不能够以先进的理念整合, 以先进的模式和方法承载, 进而以生动鲜活的方式呈现所导致的。对我国高校 而言, “教学理念相对落后、教学内容方法陈旧”一直是影响课程建设水平和人才培养效果的 “顽瘴瘨疾”。要落实 立德树人根本任务, 致力培养时代新人, 就必须推进 “课堂革命”, 从根本上改变课堂教学形态。

值得欣喜的是, 近年来, 关注教学理念和教学方法改革、关注提升教学层次、增强教学效果的老师显著增加。

《大学化学》接受的有关教学理念、教学模式、教学方法、考试方法改革的论文大幅增多。为了形成推进合力、加 强示范引领, 编辑部决定汇集出版 “教学理念和方法改革” 专刊。本专刊共汇集论文39篇, 其中涉及教学理念改革 的 10 篇, 涉及教学模式和教学方法改革的 25 篇(包括线上教学 4 篇, 混合式教学 9 篇), 涉及考试方法改革的 4 篇。希望 广大教师以这些论文为借鉴, 共同推进化学类课程教学改革再上新台阶, 再显新成效, 达成 “两性一度”, 推进课程 一流建设。

\title{
建构主义思想指导下理论课与实践课有机结合的探索
} 以大学化学类课程为例

彭一鸣 $1,{ }^{*}$, 刘英菊 ${ }^{1}$, 刘伟章 2 , 邓远富 ${ }^{3}$

1 华南农业大学材料与能源学院, 广州 510642

2 华南农业大学数学与信息学院, 广州 510642

3 华南理工大学化学与化工学院, 广州 510640

摘要: 尝试在建构主义教学思想指导下, 通过微课、 $Q Q$ 课程群、学生互助学习小组等举措对无机及分析化学课程与基 础化学实验课程进行有机结合的改革与创新。补足目前教学过程中的短板, 方便学生通过微课 “直观” 地理解理论, 掌 握实验原理。实践表明, 两门课程均获得不错的教学效果, 为继续深化课程教学改革积累了经验。

关键词: 建构主义; 无机及分析化学; 基础化学实验; 有机结合; 微课

中图分类号: G64; O6

\section{Exploration on the Combination of Theory and Practice Courses under the Guidance of Constructivism: Taking General Chemistry Courses as an Example}

Yiming Peng ${ }^{1,{ }^{*}}$, Yingju Liu ${ }^{1}$, Weizhang Liu ${ }^{2}$, Yuanfu Deng ${ }^{3}$

收稿: 2020-09-28; 录用: 2020-10-20; 网络发表：2020-12-04

“通讯作者, Email: lilypengyiming@sina.com

基金资助: 华南农业大学校级教改项目 “无机及分析化学课程中引入微课的改革与初探” (K18271); 无机及分析化学省级精品资源共享课程(粤 教高函[2015]133号)；2019年广东省质量工程项目(无机及分析化学教学团队)资助(教字[2019]95号); 国家级一流本科专业建设资助(教高厅函 [2019]46号) 


\footnotetext{
${ }^{1}$ College of Materials and Energy, South China Agricultural University, Guangzhou 510642, China.

${ }^{2}$ College of Mathematics and Informatics, South China Agricultural University, Guangzhou 510642, China.

${ }^{3}$ College of Chemistry and Chemical Engineering, South China University of Technology, Guangzhou 510640, China.
}

\begin{abstract}
Under the guidance of the concept of constructional thought, this paper attempts to reform and innovate the courses of "inorganic and analytical chemistry" and "basic chemistry laboratory" through the combination of microlectures, $Q Q$ curriculum groups and students' mutual learning groups. It makes up for the shortcomings in the current teaching processes, and makes it more conveniently for students to understand the theoretical knowledge and master the principle through the "intuitive" micro-lectures. The practice shows that good results in teaching have been achieved from these two courses, which will contribute to accumulating experience for further deepening teaching reform in the future.
\end{abstract}

Key Words: Constructivism ; Inorganic and analytical chemistry; Basic chemistry laboratory; Proper combination; Micro-lectures

无机及分析化学和基础化学实验课程均为高等农业院校大农科、生科类、林学类、兽医动物类、 理科、工科等各专业必修的专业基础课程; 并且均开设于大一上学期, 是学生入校后最早接触到的 专业相关课程, 其课程的学习质量、传递的教学理念对学生整个大学生涯都有重要引领作用。在华 南农业大学, 每年有3000多位大学新生同时学习两门课程。无机及分析化学是一门实践性很强的 课程 ${ }^{[1,2]}$, 需要学生通过实验将所学的理论知识与实践结合, 从而提高学生的动手能力、分析和解决 问题的能力。基础化学实验作为其对应的实验课程, 包含大量基础验证性实验, 对学生深入理解掌 握化学理论概念, 养成严谨求实的科学态度起到积极作用 ${ }^{[3-5]}$ 。因此无机及分析化学与基础化学实验 是一对相辅相成、密不可分的课程, 在历年大学本科教学大纲中都强调了它们的相互依存性。但是 在当前的教学模式下, 理论课与实验课在实际操作过程中却无法顺利地实现对接 ${ }^{[6]}$ 。这种脱节的教 学模式让两门课程的教与学都费时费力, 对培养学生创新性能力以及养成良好的科学素养都极为不 利，不符合新时代国家对高水平创新型人才的需求 ${ }^{[7,8]}$ 。

建构主义的最早提出者可追溯至20世纪瑞士的儿童心理学家皮亚杰, 经过柯尔伯格、斯腾伯格、 维果斯基等人的进一步完善与发展后开始运用于实际教学过程之中。建构主义近年来逐渐被国内教 育界认可并在教学实践过程中尝试运用 ${ }^{[9-11]}$ 。与过去 “灌输式” 教学方式不同, 建构主义提倡以学 生为中心, 提倡教师利用情景教学、协助、一对一等方式, 增强学生的自主能动性, 从而提高教学 效率, 提升教学效果。本文通过建构主义教学方式, 利用微课这一创新教学模式 ${ }^{[12,13]}$, 以及 $Q Q$ 学习 群等信息化手段将无机及分析化学与基础化学实验课程有机结合, 以实现理论课与实践课程双赢的 局面。

\title{
1 建构主义教学思想简介
}

建构主义教学思想核心理论为: 学生通过一定的情景, 利用必要的学习资源构建出新知识。学 生将作为学习的主体而存在; 教师帮助学生理解抽象概念, 发展和修正对问题的认识, 使学生能构 建正确的概念并深入理解。顺利实现这一既定目标, 则需要教学设计实施过程中包括 “情景” “协作” “会话” “意义建构” 四大要素。在建构主义学习环境中, 有利于学生的 “情景创设” 是教学设计中 最重要的一环; 而 “情景” “协作” 与 “会话” 三要素紧密结合是实现 “意义建构” 的重要手段 [14-16]。 在 “互联网+教育”时代，可以运用信息与通信技术来支持这四大要素的完美实现：

(1) 微课视频可以为学生创造出 “身临其境” 的 “情景” 教学方式。在理论课程中嵌入实践性微 课, 为学生提供直观了解理论知识的机会, 最大限度地还原理论知识发现总结的过程, 顺应学生的认 知规律与学习习惯, 补足目前教学过程中的短板。

(2) 建立课程学习QQ群可以规避空间时间的限制, 随时随地为学生提供协助、一对一的帮助, 
以达到最佳的学习效果, 有效实现建构主义教学思想中的 “协作”与 “会话” 环节。

\section{2 建构主义教学模式下理论课与实践课有机结合的方式}

\section{1 有机结合之 “情景” : 两门课程中引入同一微课}

微课视频内容的剪辑、编排直接决定着其质量与水平。针对理论课与实验课脱节的现况, 采用 “建构主义” 的教学思想, 以微课视频为媒介, 合理编排实现了 “理论-实验一理论” 的无缝对接。在 一个10 min左右的微课视频中, 首先介绍无机及分析化学课程中理论知识的概况; 然后衔接基础化 学实验课程中相应的实验场景; 最后画面转回到课堂部分进行知识点小结与巩固。通过微课实现了 理论与对应实验穿插在一起, 一一对应、有机结合。

\subsection{1 有效数字概念的微课}

有效数字概念微课, 大约 $10 \mathrm{~min}$ 时长: (1) 首先由教师简单介绍无机及分析化学中有效数字的定 义, 大约用时 $0.5 \mathrm{~min}$; (2) 接着画面马上切入实验部分。视频中选取了在日常生活中以及实验室较 为常见、具有代表性的一系列称量工具：家用台秤、家用电子秤、实验室电子台秤、实验室电子天 平。所有称量工具的读数部分都给予特写镜头; 然后再通过真人实操画面, 介绍各称量工具科学规 范的使用方法。学生通过观看该部分视频, 能在短短 4 min左右的时间里清晰了解到各类型实验数据 的 “来龙去脉”。(3) 画面再次转回到课堂授课模式。教师引导学生总结有效数字的概念, 分析表达 倍数关系的数字与实验数据的区别。(4) 在微课的最后部分, 带领学生理解 $\mathrm{pH}$ 值等类型数据的有数 数字判断方法。

\subsection{2 酸碱滴定分析法微课}

通过笔者制作的酸碱滴定法微课视频, 学生能直观地了解: 选择合适指示剂的目的; 整个章节 繁复计算的作用。学生在中学时代都接触过酸碱中和的概念及相应的化学方程式, 因此该视频首先 从实验现象部分切入, 再进行归纳总结上升到理论高度。(1) 首先是 $1 \mathrm{~min}$ 时长的指示剂加入前后实 验效果对比, 溶液从一直保持无色透明状态到瞬间颜色突变, 现象非常明显。(2) 接着视频分别完整 地介绍实验室中酸滴定碱的过程和碱滴定酸的过程。为了让学生有亲切感, 剪辑的视频均为上一届 学生真实实验的画面, 一共用时 $4 \mathrm{~min}$ 。(3) 画面转回到课堂授课部分。利用大约 $5 \mathrm{~min}$ 的时间, 首先 引导学生明白理论计算只是手段, 选择合适的指示剂才是酸碱滴定法的目标; 然后才介绍突跃范围 的计算方法。

\subsection{3 原电池主题微课}

原电池知识点与中学阶段的氧化还原知识点紧密相关, 但也有明显区别。中学阶段统一默认为 标准状态下, 而无机及分析化学中的原电池这一概念是研究在任意状态下的情况。为了强调这一区 别, 原电池主题微课的编排重点为展示任意状态下原电池变化实验。(1) 首先出现的画面是 $1 \mathrm{~min}$ 时 长的在标准状态下原电池基本情况的简单介绍。(2) 接着展示的是原电池在各种浓度下的实验现象, 用时 3 min。(3) 画面切换到课堂教学模式, “趁热打铁” 利用学生刚建立的认知, 引入原电池章节 中的重要知识点一一能斯特方程式, 在 $3 \mathrm{~min}$ 之内完成介绍。(4) 微课视频的最后部分, 为了激发学 生学习原电池的兴趣与热情, 效仿当前最流行的电影剪辑手法, 增加了彩蛋环节。在 $2 \mathrm{~min}$ 左右的彩 蛋环节中分别展示了利用原电池原理测量了可乐、鲜橙汁、白酸的酸度, 并发现三者的酸度非常接 近, 颠覆学生的常识性认知, 激发起学生后续学习的热情。

制作完成的微课视频同时挂在我校的无机及分析化学省级精品资源共享平台和基础化学实验省 级精品资源共享平台上, 供两门课程的师生反复观看学习。学生可以根据个人实际情况, 选择性地 利用微课对理论课程与实验课程进行预习和复习。微课视频针对性地帮助学生深入理解理论知识, 明确掌握实验原理, 减少教师重复工作, 提升学生的学习效率, 让实验课程真正发挥出培养学生动 手与创新能力的功能, 实现了理论课与实验课两者的有机融合, 具体情况如图1所示。 


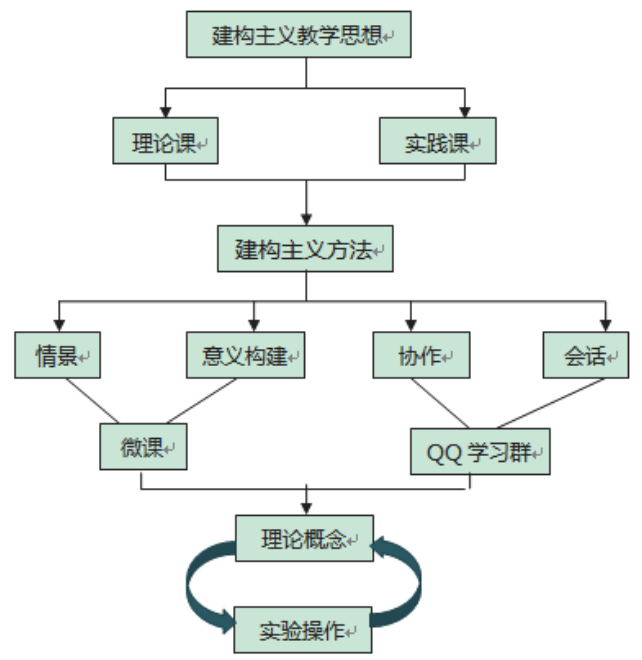

图1＼cjkstart建构主义思想指导下理论课与实践课有机结合示意图

\section{2 有机结合之 “会话” : 两门课程中引入同一学习QQ群}

从早期的广播电视大学的经验中可以得出, 学习过程中得不到反馈则教学效果差。根据建构主义 教学思想, 建立课程学习 $\mathrm{QQ}$ 群, 并邀请无机及分析化学与基础化学实验两门课程的教师加入QQ群, 为 学生随时解答与课程相关的理论、实验部分各类疑问。

\subsection{1 有效利用QQ群私聊功能}

由于学生基础不同, 对同一知识点的理解与掌握程度各不相同, 无论是微课视频还是翻转课堂 都很难做到满足所有学生的学习需求, QQ学习群的私聊功能应运而生。QQ群的私聊功能保障了教 师、学生 “一对一” 针对性辅导, 有的放矢事半功倍。

例如无机及分析化学理论课程介绍完酸碱指示剂的选择方法后, 学生会进行相应的实验。在QQ 群私聊里, 学生有些还在追问突跃范围的计算方法; 部分学生已经在为自己想要做的印证实验细节 而提问了。

再如无机及分析化学热力学章节中标准状态下转折温度的计算公式为 $T=\Delta_{\mathrm{r}} H_{\mathrm{m}}^{\ominus} / \Delta_{\mathrm{r}} S_{\mathrm{m}}^{\ominus}$, 教师在课 堂上通常会重点强调焓变值与熵变值单位的统一。通过QQ课程群私聊功能, 对学生个别辅导发现有 部分同学的纠结: 该计算公式中焓变值与熵变值的符号是否需要统一, 用以保证计算所得的热力学 转折温度永远大于零。

\subsection{2 有效利用QQ群群聊功能}

无机及分析化学课程中有一些公认的知识难点, 例如有效数字概念中 $\mathrm{pH}$ 值的有效数字位数的确 定。笔者的教改尝试如下: (1) 上课之前, 教师在QQ群中首先布置学习任务: 根据微课视频以及书 中的运算法则, 学会将氢离子浓度换算成相应的 $\mathrm{pH}$ 值。然后鼓励学生之间互问互答, 确保每位学生 都掌握该运算方法。(2) 在课堂上先组织学生再次观看有效数字的微课视频, 接着给学生 2 min分组 讨论 $\mathrm{pH}$ 值等类型数据的有数数字判断方法, 将小组意见发布在QQ课程群中。(3) 教师总结并布置相 关小练习, 鼓励学生课后在QQ课程学习群继续讨论。

学生之间互问互答、积极讨论, 最后再由教师进行科学总结最能激发学生的参与热情, 提高学 习效率。再如基础化学实验课程中的操作实验 - $\mathrm{CuSO}_{4} \cdot 5 \mathrm{H}_{2} \mathrm{O}$ 结晶, 该实验的难点在于是否能得到 晶体产品。实验结束后, 一位得到絮状沉淀的学生在QQ群里首先发问, 实验课教师因势引导学生在 群里讲述可能存在问题的实验步骤。通过充分开放式的讨论, 实验课程教师归纳总结出实验注意事 项, 最后再由理论课程教师引出无机及分析化学课程中有关沉淀部分的理论知识点, 完成了 “理论实验-理论” 的全过程, 获得不错的教学效果。 


\section{3 有机结合之 “协作” : 建立学生间的互助学习小组}

课程微课改革的成效与学生的配合紧密相关, 建立学生间的学习互助组可以督促每一位学生都 积极参与。笔者让学生针对无机及分析化学课程自愿结成互助小组, 每组大约 6 到 10 人左右, 并自选 出小组长; 针对基础化学实验课程设立两人实验小组。为了避免学习小组流于形式, 充分调动学生 的参与积极性, 在学校教务处的帮助下, 加大了平时成绩在期末总评成绩中的权重。无机及分析化 学课程中平时成绩占比为 $40 \%$, 期末考试卷面成绩占比为 $60 \%$; 基础化学实验课程中平时成绩占比高 达 $80 \%$ 。其次为了避免平时成绩流于形式, “量身定制” 了相应的平时成绩评分细则:

(1) 针对无机及分析化学课程该细则分为两类: 一大类为学习小组评分, 组内所有组员得分相 同, 包括: (1) 设立随堂测验制度, 一学期进行三次, 由学习小组成员共同完成, 满分为9分; (2) 课 前小组自行组织观看微课视频, 并完成小作业, 满分为 9 分。(3) 将期末考试卷中分值为 5 分的简答题 出题权交给各学习小组。要求各小组根据课本里介绍过的知识点, 理论联系实践出一道简答题, 满 分为 5 分; 其中的佼佼者可入选课程期末考试题库或直接出现在当年的期末考卷上。另一大类为个人 自得分数, 包括: (1) 个人出勤率, 由小组长记录, 满分 5 分; (2) 课后作业, 主要考核是否按时完成 并上交, 不记录正确率, 由小组长负责登记, 满分12分。

（2）针对基础化学实验课程包括: (1) 观看微课视频或自己翻阅资料书写预习报告, 满分 20分; (2) 实验时小组同学必须协作, 两位同学该项分数保持一致, 满分 50 分; (3) 数据处理与结果讨论, 满分30分。

\section{3 理论课与实践课有机结合教学模式的效果反馈}

\section{1 期末考试成绩反馈无机及分析化学教学效果}

\subsection{1 期末考试不及格率比较}

无机及分析化学课程一直是我校考试通过率较低、整体优良率偏低的课程, 多年来被学生戏称 为 “双繁”之一。无机及分析化学与基础化学实验课程进行有机结合教改后, 经过一个学期的探索 与实践, 取得了可喜的成果。在相同大类专业学生考核要求和题型均一致的情况下, 对比近几年我 校无机及分析化学课程期末考试卷面成绩, 可以看出引入课程信息化改革之后学生的整体考试成绩 有了较大的提升。以生科大类的学生为例, 近三年该课程的期末考试卷面成绩不及格率都在 $10 \%$ 以 上, 并且逐年上升大有越演越烈之势，2018级学生的不及格率已经达到 “触目惊心” 的 $26.3 \%$ 。开启 信息化教学改革之后, 经过师生的共同努力, 2019 级学生的不及格率仅为 $4.9 \%$, 成功的下降到 $5 \%$ 以 下的超低区间。

\subsection{2 教改前后期末卷面成绩高分段比较}

从表 1 和表 2 的数据可以看出, 无机及分析化学课程嵌入实践性微课教学改革后, 充分调动了学 生的学习能动性, 课程学习中历年来固有的一些 “顽疾” 被清除了, 学习成绩整体显著提升, 年级 平均优良率从 $11.5 \%$ 飞跃到 $49.2 \%$ 。

表1＼cjkstart教改前生科大类学生期末卷面成绩高分段统计表

\begin{tabular}{cccccc}
\hline 班级 & 学生总人数 & 90 分以上人数 & 最高分 & 各班优良率 & 平均优良率 \\
\hline 生科大类1 & 29 & 0 & 82.5 & $10.3 \%$ & $11.5 \%$ \\
生科大类2 & 30 & 0 & 86 & $3.3 \%$ & \\
生科大类3 & 30 & 0 & 87 & $20 \%$ & \\
生科大类4 & 17 & 0 & 81.5 & $5.8 \%$ & \\
生科大类5 & 30 & 0 & 84.5 & $13.3 \%$ & \\
生科大类6 & 29 & 0 & 88.5 & $13.7 \%$ & \\
\hline
\end{tabular}


大学 化 学 Univ. Chem. 2021, 36 (7), 2009063 (6 of 7)

表2 教改后生科大类学生期末卷面成绩高分段统计表

\begin{tabular}{cccccc}
\hline 班级 & 学生总人数 & 90分以上人数 & 最高分 & 各班优良率 & 平均优良率 \\
\hline 生科大类1 & 28 & 0 & 88.5 & $32.1 \%$ & $49.2 \%$ \\
生科大类2 & 28 & 3 & 94.5 & $25 \%$ & \\
生科大类3 & 23 & 3 & 94.5 & $26.1 \%$ & \\
生科大类4 & 30 & 10 & 98 & $70 \%$ & \\
生科大类5 & 30 & 11 & 96 & $73.3 \%$ & \\
生科大类6 & 29 & 5 & 93 & $48.3 \%$ & \\
生科大类7 & 31 & 8 & 97 & $61.2 \%$ & \\
\hline
\end{tabular}

\section{2 基础化学实验教学效果}

\subsection{1 教师意见反馈}

在学期末的总结座谈会上, 基础化学实验课程组的教师也反馈: 教改前学生的主观能动性发挥 不够; 不能有效理解实验原理; 机械照搬实验步骤; 对实验结论没有科学认识; 学生实验报告雷同 性高; 更有甚者, 实验步骤混乱, 实验药品添加随意。两门课程有机结合改革后, 学生最明显的变 化是: 实验课程的学习态度端正了, 积极主动性提高, 规避了不少往届学生常见的错误。学生不再 是被动机械照搬书本实验, 而是目标明确地验证前人科研成果; 并且会主动对异常数据进行理性客 观的分析讨论。

\subsection{2 期末成绩反馈}

从表3中可以看出理论课与实践课进行结合教改后, 学生实验成绩整体提高, 优秀率显著增加, 学习态度与学习效果明显改善。

表3 基础化学实验期末成绩教改前后对比

\begin{tabular}{cccccc}
\hline 教改 & 优秀(90分以上) & 良好(80-90分) & 中等(70-79分) & 及格(60-69分) & 不及格 \\
\hline 前 & $41.38 \%$ & $48.28 \%$ & $10.34 \%$ & 0 & 0 \\
后 & $60.71 \%$ & $35.71 \%$ & $3.57 \%$ & 0 & 0 \\
\hline
\end{tabular}

\section{3 学生意见反馈}

两门课程结束后, 学生对该课程教学综合评价为93.21分。学生在课程留言处踊跃留言, 获点赞 最多的留言为 “第一次发现实验课程的重要性, 还想多做一些实验”。课程问卷调查显示: 92.59\%的 学生对课程总体满意, 尤其是新型平时成绩考核办法与建立课程 $\mathrm{QQ}$ 学习群两项好评度占绝对优势。 根据学生积极正面的反馈信息可以看出, 理论课程与实践课程有机结合这一举措, 顺应学生的主流 学习需求。

\section{4 结语}

利用现代网络信息手段, 在建构主义思想指导下, 将无机及分析化学与基础化学实验课程有机 结合的教学改革思路, 在我校农科学生中的尝试取得了预期的效果, 得到了大多数学生的肯定与支 持, 为继续深化课程教学改革积累了经验。

在目前 “互联网+教育” 的信息时代大背景下，依据 “建构主义” 教学思想设计了无机及分析化 学与基础化学实验课程有机结合的创新教学模式, 补齐了目前理论教材方面普遍存在的短板, 实现 了 “理论-实验-理论” 的教学构思, 确保理论课与实验课程的相互融合促进。信息化教学改革有效地 改善了暮气沉沉的理论课堂气氛, 满足了学生个性化学习的需要; 让实验课程也真正发挥出其培养 新时代创新型人才的作用。 
[1] 倪春林, 刘英菊, 罗志刚, 卢其明, 杨乐敏. 实验室科学, 2012, 15 (3), 167.

[2] 杨沙沙, 纪恩, 黄森. 教育教学论坛, 2013, No. 50, 82 .

[3] 宋继梅, 杨捷, 吴振玉, 吴杰颖, 田玉鹏. 实验室研究与探索, 2011, 30 (1), 106.

[4] 宰建陶, 陈虹锦. 实验室研究与探索, 2018, 37 (9), 227.

[5] 李大枝, 范传刚. 实验技术与管理, 2019, 36 (11), 191.

[6] 杨巍. 化学教育, 2016, 37 (16), 73.

[7] 袁腾, 刘英菊, 陈燕舞, 汤日元, 倪春林. 实验室研究与探索, 2017, 36 (6), 209.

[8] 袁腾, 林楠, 汤日元, 刘海峰, 杨卓鸿. 实验技术与管理, 2019, 36 (5), 48.

[9] 韩清娟, 张梦军, 刘明. 现代医药卫生, 2020, 36 (12), 1923.

[10] 赵月春, 莫测辉. 高等农业教育, 2007, 189 (3), 56.

[11] 唐小兰, 刘英菊. 大学化学, 2016, 31 (7), 34.

[12] 胡铁生, 周晓清. 现代教育技术, 2014, 24 (2), 5.

[13] 张召香, 张飞, 王卫, 林洁华. 化学教育, 2020,41 (10), 35.

[14] Spector, J. M.; Merrill, M. D.; Elen, J.; Bishop, M. J. 教育传播与技术研究手册. 第4版. 任友群, 焦建利, 刘美风, 汪琼, 顾小清, 闻寒冰, 译. 上海: 华东师范大学出版社, 2015: 18-19.

[15] 何克抗. 电化教育研究, 2013, 243 (7), 24.

[16] 何克抗. 电化教育研究, 2013, 246 (10), 29 . 